had two to four relapses within 3 months and 6 years following improvement after the initial episode. These patients had received minimal or no immunotherapy for the initial illness. Good clinical outcome correlated with decreased NMDA receptor antibody levels and were associated with early immunotherapies. Progression in relapse cases was in two stages, initial neuropsychiatric symptoms and seizures, followed later by onset of movement disorders, impaired consciousness and dysautonomia. Early features are associated with CSF lymphocytosis, and later features with appearance of oligoclonal bands. (Irani SR et al. Brain 2010;133:1655-1667).

\title{
MMR-VARICELLA VACCINE AND RISK OF FEBRILE SEIZURES
}

Researchers from the Kaiser Permanente Vaccine Study Center, Oakland, CA, other centers in the US, and Centers for Disease Control and Prevention, Atlanta. GA, using 2000-2008 Vaccine Safety Datalink data, compared the risk of febrile seizures among children aged 12 to 23 months after MMRV and separate MMR + varicella vaccines. Analyses included Poisson regression and supplementary regressions incorporating chart-review results. Seizure and fever incidence significantly peaked 7 to 10 days after vaccination with all measles-containing vaccines but not after varicella vaccination alone. The relative risk of seizures was $7.6(\mathrm{P}<0.0001)$ after $\mathrm{MMRV}$ combined vaccination, $4.0(\mathrm{P}<0.0001)$ after $\mathrm{MMR}+$ varicella separate vaccinations, and $3.7(\mathrm{P}<0.0001)$ after MMR vaccination alone. No seizure peak occurred after varicella vaccination alone. Rates for seizures during days 7 to 10 were $84.6 / 1000$ person-years after MMRV, 42.2 seizures per 1000 person-years after MMR + varicella, and 26.4/1000 after MMR alone. Unadjusted rates were 8 times higher for MMRV and 4 and 3.5 times higher for MMR + varicella and MMR vaccination alone, respectively. Outpatient fever visits sharply increased during days 7 to 10 , most actively after MMRV. The proportion of children with post-vaccination febrile seizures with a history of seizures was similar for MMRV $(16 \%)$ and MMR + varicella vaccines $(22 \%)[\mathrm{P}=0.21]$. Of those with febrile seizures, family history was positive for seizures among $30 \%$ of MMRV recipients vs $29 \%$ of MMR + varicella vaccines $(\mathrm{P}=0.90)$. The use of MMRV vaccine instead of separate MMR + varicella vaccines approximately doubles the risk for fever and febrile seizures. Providers who use the combination vaccine should notify parents of this increased risk of febrile seizures. (Klein NP, Fireman B, Yih WK, et al. Measles-mumpsrubella-varicella combination vaccine and the risk of febrile seizures. Pediatrics July 2010;126:e1-e8). (Respond: Nicola P Klein MD PhD, Kaiser Permanente Vaccine Study Center, 1 Kaiser Plaza, Oakland, CA 94612. E-mail: nicola.klein@kp.org).

COMMENT. The risk of fever and febrile seizures is increased 7 to 10 days following all measles-containing vaccines, and the risk after the combination MMVR vaccine is double that associated with separate MMR + varicella vaccines. Varicella vaccine alone does not increase febrile seizure risk.

Diphtheria-tetanus acellular pertussis vaccine (DTaP) in children aged 6 weeks to 23 months showed no increased risk of seizures within 0 to 3 days after vaccination, in a retrospective study from 1997 through 2006 conducted by 7 managed care organizations and the Vaccine Safety Datalink Team using risk-interval cohort and self-controlled case 
series analyses. (Huang W-T, Gargiullo PM, Broder KR, et al. Pediatrics August 2010;126:e263-e269). By contrast published studies report a relative risk of 3.3 for febrile seizures within 0-3 days of DTP whole-cell pertussis vaccine (RR 5.7 on the same day as vaccination). (Barlow WE et al. New Engl J Med 2001;345:656-661).

\section{YIELD OF LUMBAR PUNCTURE WITH FIRST COMPLEX FEBRILE SEIZURE}

Researchers in emergency medicine and pediatric neurology at Children's Hospital Boston, MA, assessed the rate of acute bacterial meningitis (ABM) among 526 children (56\% male) aged 6 to 60 months (median age 17 months) who were evaluated in the ED between 1995 and 2008 for a first complex febrile seizure (CFS). Ninety patients (17\%) had a previous simple febrile seizure. Of the total with CFS, $340(64 \%)$ had a lumbar puncture; $14(2.7 \%)$ had CSF pleocytosis. Three patients $(0.9 \%)$ had ABM; 2 had csf pleocytosis, diplococci, and a positive culture for Streptococcus pneumoniae. Of the 2 with confirmed ABM, 1 aged 4 years was found unresponsive at presentation and had posturing of one arm and anisocoria; the other aged 11 months was sleepy and on examination, appeared flaccid, toxic, with bulging fontanel, nuchal rigidity and apnea. The third child had failed the LP test (no csf cell count), she appeared well at presentation, but her blood culture grew $S$ pneumoniae and she was treated as suspected ABM. None of the patients discharged without LP returned with a diagnosis of ABM. The decision to perform LP with a first CFS should be based on clinical suspicion and additional signs and symptoms suggestive of meningitis. ABM in a child presenting with CFS is uncommon $(<1 \%)$, and the risk is particularly low with CFSs manifested by 2 brief nonfocal seizures in 24 hours. (Kimia A, Ben-Joseph EP, Rudloe T, et al. Yield of lumbar puncture among children who present with their first complex febrile seizure. Pediatrics July 2010;126:62-69). (Respond:Amir Kimia MD, Division of Emergency Medicine, Children's Hospital Boston, 300 Longwood Ave, Boston, MA 02115. E-mail: amir.kimia@childrens.harvard.edu).

COMMENT. The rate of seizure among children with acute bacterial meningitis is reported at $12 \%$ (Rosman NP et al. 1985) to $27 \%$ (Rosenberg NM et al. 1992). In the above series of patients diagnosed with a first complex febrile seizure, the rate of bacterial meningitis among those having an LP was quoted at $0.9 \%(0.6 \%$ in the total group). Routine LP is obviously inappropriate in all children with a complex febrile seizure, especially in those with short nonfocal seizures repeated in 24 hours. The 2 patients with confirmed ABM $(0.5 \%)$ in the Boston series had symptoms and signs of meningitis other than seizure. Furthermore, the description of presenting features was not convincing for a diagnosis of seizure.

Investigation of infections causing febrile seizures. In a study of 100 consecutive febrile seizures, LP was performed in 14 patients ( 11 complex seizures and 3 simple). Clinical suspicion and complex seizures were the principal indications for LP, and not age. Viral infection was the most common cause of fever, and bacterial infection was infrequent. Bacterial cultures performed in all 100 patients were positive in only $5 \%$, none from cerebrospinal fluid. Rapid viral testing and diagnosis would result in less emphasis on need for LP and a reduction in empiric antibacterial treatment. Complex 